\title{
CALCULATION OF THE TIDE CORRECTION USED IN GRAVIMETRY
}

\author{
Rogério Rodrigues Amarante and Jorge Luiz Alves Trabanco
}

\begin{abstract}
In gravimetric surveys, the determination of the gravity at a specific point depends on field measurements and on the removal of the influence of the tide. With the current development of more sensitive gravimeters, a review of the tide correction calculation process applied in gravimetry is necessary because values that were once considered negligible may represent noise above the resolution of the new equipment. Much used until the present, the formulas shown in the study by Longman (1959) were elaborated with constants (distances, masses, ellipsoidal parameters, and orbital values) that can now be calculated more accurately, as well as conveniently rounded values for the time. The deformation of the crust caused by the terrestrial tide is another important factor that significantly affects the tide correction calculation results, and the Earth's heterogeneity causes this deformation to differ between regions. Much effort has been made to map different regions with the objective of regionalizing the gravimetric factor; however, computer programs and even modern automated equipment still use fixed values. Therefore this paper aims to present a review that quantifies the impacts on the calculation of the tide correction considering parameters not yet used.
\end{abstract}

Keywords: earth tide correction, gravimetric factor, gravimetry, gravimetric surveys.

RESUMO. Em levantamentos gravimétricos a determinação da gravidade de um ponto depende de medições em campo, bem como da remoção da influência da maré. Com o desenvolvimento atual de gravímetros mais sensíveis, torna-se necessário uma revisão do processo de cálculo da correção da maré aplicado na gravimetria, pois valores antes considerados desprezíveis podem representar ruídos acima da resolução dos novos equipamentos. Muito utilizada até o presente, às fórmulas apresentadas no trabalho de Longman (1959), foram elaboradas com constantes (distâncias, massas, parâmetros elipsoidais, valores orbitais) hoje atualmente reformuladas, bem como valores convenientemente arredondados para época. Outro ponto importante é a deformação causada na crosta pela maré terrestre que afeta significativamente o resultado do cálculo da correção da maré, e que a heterogeneidade da Terra faz esta deformação ser diferente entre as regiões. Muito esforço tem sido realizado no sentido de mapear diferentes regiões objetivando uma regionalização do fator gravimétrico, contudo softwares usados para redução gravimétrica e até mesmos equipamentos automatizados ainda utilizam valores fixos. Portanto este trabalho se propõe apresentar uma revisão, quantificando o impacto no resultado de fatores e influências ainda não considerados no cálculo da correção da Maré.

Palavras-chave: correção da maré terrestre, fator gravimétrico, gravimetria, levantamento gravimétrico. 


\section{INTRODUCTION}

The vector of the gravitational force $\vec{g}$ at a specific point $P$ on the Earth's surface is the result of the forces of attraction of the terrestrial mass $\vec{F}$ and the centrifugal force $\vec{C}$ applied to the mass of the body stationed at that point. This gravitational acceleration is expressed in $\mathrm{m} / \mathrm{s}^{2}$ or Newton ${ }^{1}$ by the $\mathrm{SI}$ (International System of Units). For gravimetrics, however, the Gal (Si, 2006) is used in homage to Galileo Galilei² (Gemael, 1985).

The law of gravity proposed by Newton in 1687 states that any particle of mass $m_{1}$ in the universe attracts another mass $m_{2}$ with a force that depends on the product of the two masses and the inverse of the square of the distance $d$ between them, as shown by function 1:

$$
\vec{F}=G \frac{m_{1} m_{2}}{d^{2}}
$$

where $G$ is gravitational constant.

It is noteworthy that Newton's Law is theoretical and refers to particles. Its elucidation is still unknown, despite the fact that this law can be observed and proven (Gemael, 2002).

Given that the Earth spins around its axis with an angular velocity $\omega$, there is a centrifugal force $\vec{C}$ that has a maximum value of approximately $0.3 \%$ of gravity at the equator and that is zero at the poles. Gravity $\vec{g}$ is the resultant vector between the attractive and centrifugal forces, according to Eq. (2) (Fig. 1).

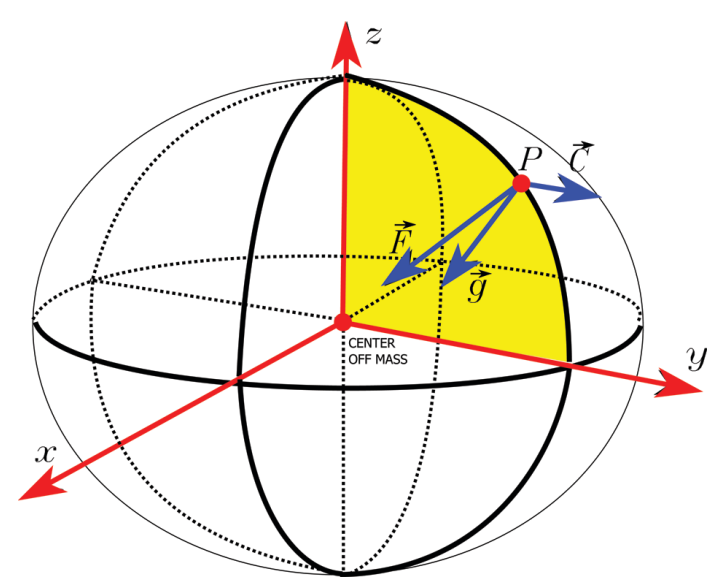

Figure 1 - Vector components of gravity. Figure adapted from Gemael (2002).

$$
\vec{g}=\vec{F}+\vec{C}
$$

where, without considering flattening of the ellipsoid (Gemael, 2002):

$$
\vec{C}=\omega^{2} r
$$

adopting the values:

$$
\begin{array}{ll}
\omega & =7.292115 \times 10^{-5} \mathrm{rad} \mathrm{s}^{-1} \text { (Petit \& Luzum, 2010); } \\
r & =R \cos \varphi ; \\
R & =\left(a^{2} b\right)^{1 / 3} \\
\varphi & \text { latitude of a point } P \text { on the surface of the Earth; } \\
r & \text { is the distance } P \text { to the center of the Earth; } \\
a & \text { equatorial radius of the Earth; } \\
b & \text { polar radius of the Earth; } \\
\omega & \text { angular velocity of the Earth. }
\end{array}
$$

Considering an ellipsoidal model, the theoretical gravity, which is the normal gravity at the level of the ellipsoid derived in numerical form from the Somigliana formula, would be (Moritz, 1980):

$$
\gamma=\gamma_{e} \frac{1+k \sin ^{2} \varphi}{\sqrt{1-e^{2} \sin ^{2} \varphi}}
$$

adopting the values:

$\gamma_{e}=978,032.67715 \mathrm{mGal}$ (Torge, 2001);

$\gamma_{p}=983,218.63685 \mathrm{mGal}$ (Torge, 2001);

$$
\begin{aligned}
f= & \text { 1/298.257222101 SIRGAS } 2000 \text { reference system, GRS80 } \\
& \text { ellipsoid (IBGE, 2005); }
\end{aligned}
$$

where:

$$
\begin{gathered}
k=\frac{b \gamma_{p}}{a \gamma_{e}}-1 \\
b=a(1-f) \\
e^{2}=\frac{a^{2}-b^{2}}{a^{2}}
\end{gathered}
$$

with:

$\gamma \quad$ normal acceleration of gravity (theoretical gravity);

$\gamma_{e} \quad$ normal acceleration of gravity at the equator line;

$\gamma_{p} \quad$ normal acceleration of gravity at the poles;

$f \quad$ ellipsoidal flattening;

$b$ minor semiaxis;

$e^{2} \quad$ square of the primary eccentricity.

The true gravimetric potential at points on the Earth cannot be determined with a simple mathematical equation because the heterogeneity and density variation of the terrestrial body make the integration impossible, thereby necessitating the measurement of values for $\vec{g}$ to create models (Gemael, 1985).

\footnotetext{
${ }^{1}$ Issac Newton (1642-1727): English physicist, mathematician, astronomer, and natural philosopher

${ }^{2}$ Galileo Galilei (1564-1642): Italian physicist, mathematician, astronomer, and philosopher
} 


\section{Gravimetry and equipment}

Gravimetry is an area of physical geodesy that has the objective of measuring the gravitational intensity and/or the gradient of gravity with terrestrial methods or methods near the Earth's surface. Gravimetric measurements can be performed using "absolute" or "relative" techniques (Torge, 2001).

Determining the value of gravity $\vec{g}$ is important because, by calculating its anomalies and it is possible to determine the undulations $\mathrm{N}$ of the geoid using the Stokes' integral, the terrestrial flattening with the Clairaut ${ }^{3}$ theorem, and the deviation from the vertical with the Vening-Meinesz ${ }^{4}$ formulas (Gemael, 1985).

Gravimetrics can be applied in geophysical investigations to estimate the density of rocks and to determine geoidal undulations; furthermore, gravimetrics can be employed in geodynamic studies, in which temporal changes of the terrestrial gravitational field are monitored.

\section{Absolute methods for measuring gravity}

Absolute gravity measurements determine $\vec{g}$ using the fundamentals of gravitational acceleration, measurement of time, and displacement. The measurement can be performed using the pendulum method, introduced by Galileo Galilei, which is currently not used, or by the free fall method (Torge, 2001; Gemael, 2002), which became viable with the technological advance of chronometers, sensors, and electronics.

The pendulum method presented in Figure 2 can be easily calculated using Eq. (8).

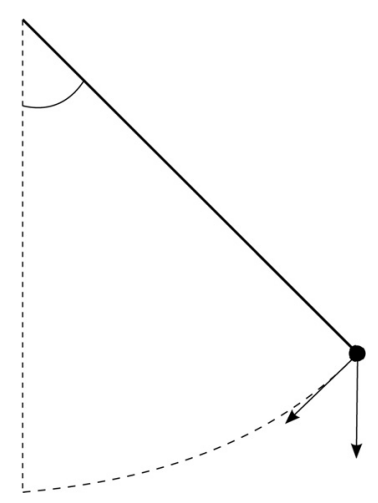

Figure 2 - Pendulum determination. Figure adapted from Gemael (2002).

$$
t=2 \pi \sqrt{\frac{l}{g}\left(1+\frac{\sin \alpha^{2}}{16}\right)}
$$

with:

\footnotetext{
${ }^{3}$ Alexis Claude de Clairaut (1713-1765): French mathematician

${ }^{4}$ Felix Andries Vening Meinesz (1887-1966): Dutch geophysicist and geodesist
}

$t \quad$ is the period in seconds necessary for the movement performed by the pendulum to repeat itself;

$l \quad$ is the length of the pendulum in meters;

$\alpha$ is the angle formed between the vertical and the initial position of the pendulum;

$g$ is gravitational acceleration.

The free fall method for determining gravity (Fig. 3) is based on the principle of accelerating movement shown in Eq. (9), which can be simplified according to formula 10 (Gemael, 2002).

$$
\begin{gathered}
x=x_{0}+v_{0} t+\frac{1}{2} g t^{2} \\
g=2\left(\frac{\left(x_{3}-x_{1}\right)\left(t_{2}-t_{1}\right)-\left(x_{2}-x_{1}\right)\left(t_{3}-t_{1}\right)}{\left(t_{3}-t_{1}\right)\left(t_{3}-t_{2}\right)\left(t_{2}-t_{1}\right)}\right)
\end{gathered}
$$

where:

$$
\begin{aligned}
& -t_{1} ; t_{2} ; t_{3} \text { are time intervals counted from } t=0 ; \\
& -x_{1} ; x_{2} ; x_{3} \text { are the distances moved in the intervals } \\
& t_{1} ; t_{2} ; t_{3} \text { respectively. }
\end{aligned}
$$

Modern instruments for absolute measurement by the free fall method are accurate; however, these machines are costly, complex, difficult to transport, and difficult to set up on irregular or unstable ground. The basic function of this type of equipment is to measure the position at several instants in time for a body in free fall in a vacuum chamber.

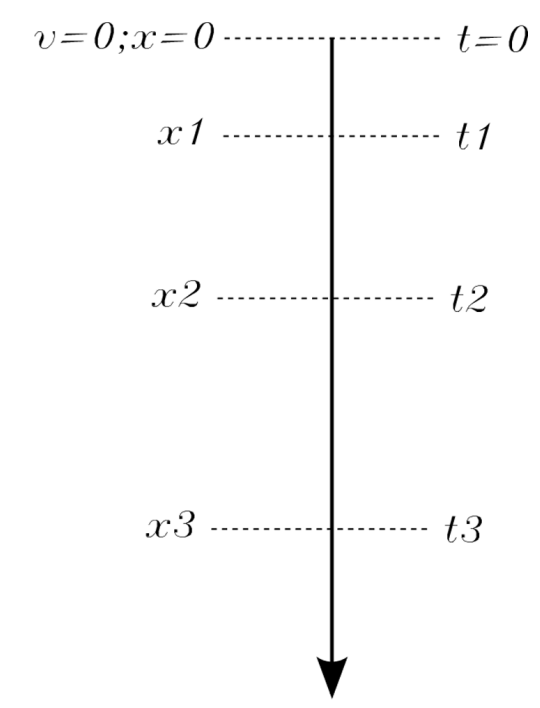

Figure 3 - Determination with the free fall method. Figure adapted from Gemael (2002). 


\section{Relative methods for measuring gravity}

Relative methods use equipment to measure the difference between the readings at one station versus another. The determination of $\vec{g}$ at a given point depends on the existence of a gravimetric station with a previously known acceleration value, from which the result can be calculated by summing the difference determined between the stations. The functioning principle is based on the weight of a body equilibrated by a normally elastic inverse force, provided by a spring in an environment with a constant temperature, to maintain the same coefficient of elasticity between the measurements. The differences in $\mathrm{mGal}$ are measured on a graduated scale, and the results are parameterized according to a calibration table provided by the manufacturer. One functioning scheme of the LaCoste and Romberg (L\&R) model G gravimeter can be observed in Figure 4.

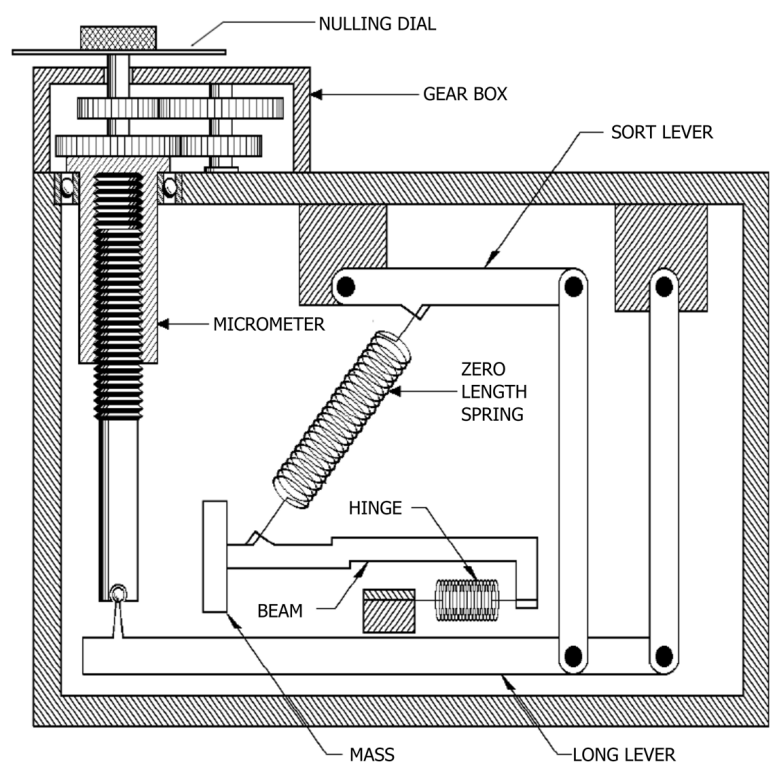

Figure 4 -Schematic section of the LaCoste and Romberg model G\&D gravimeter (LaCoste \& Romberg, 2004).

The emergence of relative measuring equipment revolutionized gravimetry, as it permitted the rapid densification of gravimetric networks due to the reduced size, ease of use, possibility of installation in diverse environments, and high productivity (Gemael, 2002).

In contrast with the absolute equipment, a common characteristic of mechanical elastic equipment and one of its primary inconveniences is the existence of a phenomenon known as drift, which should be considered in gravimetric calculations. To illustrate, a comparison between a piece of equipment based on free fall and another machine with an elastic system is shown in Figure 5. There are two different types of drift: statistical and dynamic. The first occurs when the elastic system is altered in a state of rest; during brief periods, the variation is normally small. However, surveying one circuit often takes days; therefore, it is inevitable that the team will break for meals and rest. Dynamic drift results from deformation of the elastic system, which occurs over time and from movements that occur during its displacement in the circuit in which the work is performed. The time limit is a characteristic that varies as a function of the model and brand of the equipment and is determined from the period necessary for the drift to be treated as linear.

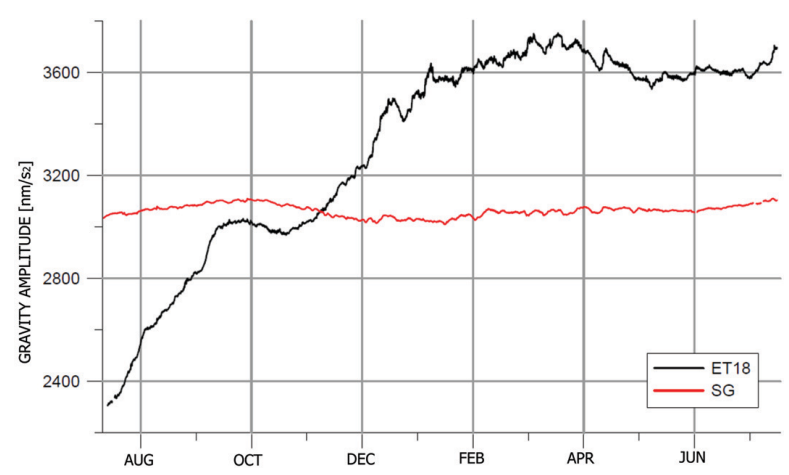

Figure $\mathbf{5}$ - Drift of the relative ET18 and absolute SG gravimeters (Hegewald et al., 2010).

Some devices (e.g., the L\&R gravimeter) depend on more extensive work to derive the results. Other more modern devices that have onboard electronics are more precise and handle the data better, further facilitating their use - as in the CG-5 Scintrex Autograv gravimeter, which is displayed in Figure 6.

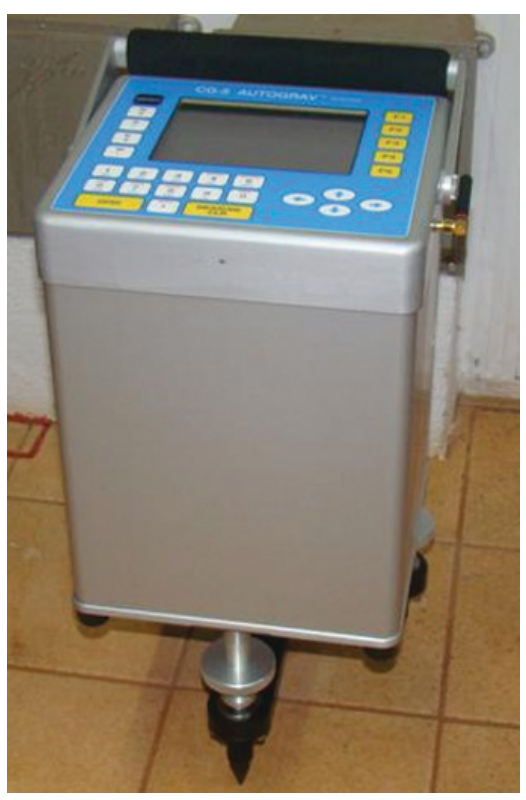

Figure 6 - CG-5 Scintrex Autograv gravimeter. 


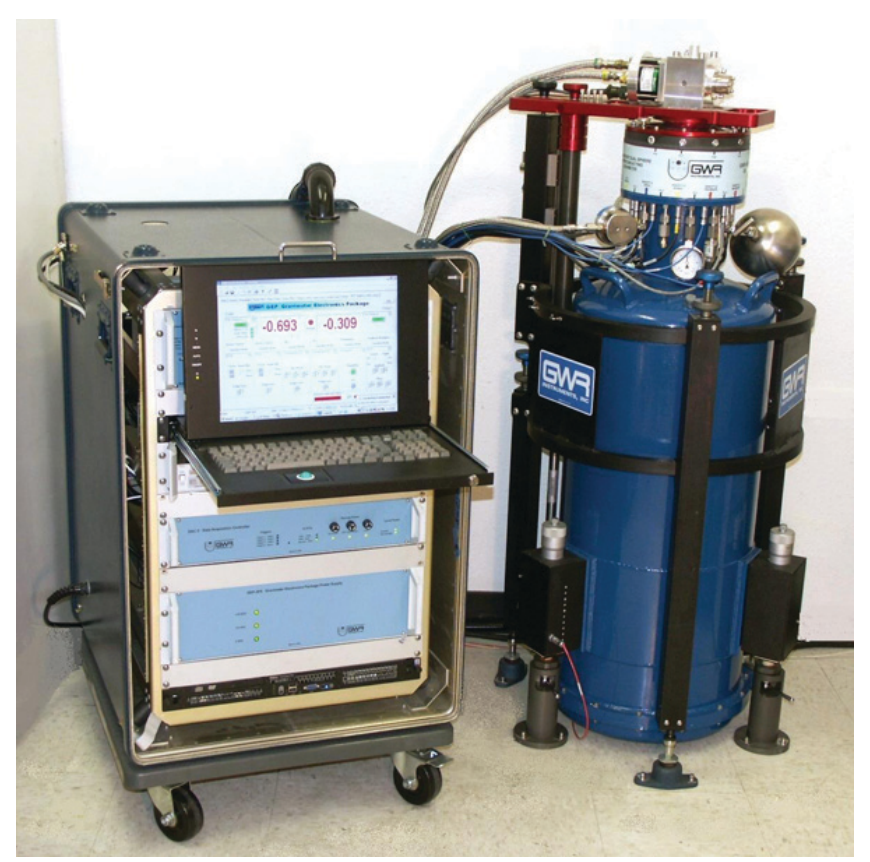

Figure 7 - OSG superconductor gravimeter (GRW, 2011).

The CG-5 is part of a new generation of relative gravimeters with microprocessors, which function in the range of $8000 \mathrm{mGals}$ with a resolution of $1 \mu \mathrm{Gal}$, without the need to be reinitiated. Unlike mechanical equipment such as the model $G$ from $L \& R$, the CG-5 does not record isolated measurements made by one operator but rather samples in pre-defined intervals or in a continuous manner at a frequency of $6 \mathrm{~Hz}$. That is, a machine that is configured to make three measurements of 1 minute each will store in its memory $(6 \mathrm{~Hz})(60 \mathrm{~s})(3)=1080$ readings in a series of measurements of one station. The fact that this gravimeter makes multiple measurements per second permits the incorporation of a seismic filter. The possibility of storing the collected data in its 12 Megabyte memory, in addition to saving work, avoids many possible errors, such as performing an inaccurate reading of the graduation from the dial, dictating the wrong value to the recorder, writing incorrectly in the field notebook, reading incorrectly from the field notebook when transcribing the data to a computer program, or even typing incorrectly. To facilitate its use, the equipment has a graphic screen, a keyboard, and a user-friendly interface. The machine also has protection against temperature changes, pressure variations, and variations caused by magnetic fields. Another important characteristic of the CG-5 is a small drift, which results from the operational environment of the elastic quartz system in conjunction with the correction by the software that provides results in real time with long-term drift and 0.02 mGals per day (Scintrex, 2008).
Another type of gravimeter is the superconducting gravimeter, shown in Figure 7. Its functioning principle involves the levitation of a spherical specimen by a magnetic field generated by coils. The sphere moves up and down in response to changes in gravity; therefore, the voltage is altered automatically, with the objective of maintaining it in equilibrium and moving the specimen to the correct position. This applied voltage is parameterized on a scale to provide the gravity changes. Superconductor gravimeters can achieve sensitivities of $1 \mathrm{nGal}$ (nanogal) in their measurements (GRW, 2011).

\section{TIDE}

The value of $\vec{g}$ must be determined from the attractive $\vec{F}$ and centrifugal $\vec{C}$ forces, and the other perturbations must be eliminated, including the tide correction $C_{g}$, which is also called the gravimetric correction. The essential elements of physics for understanding tides are contained in Newton's laws of dynamics and conservation of mass (Pugh, 1987).

The total gravitational attraction between two large masses such as the Earth and the Moon, as well as their proximity, makes this set a system. All other bodies in space also attract each other; however, because the distances are so large, the forces are extremely small, with the exception of the Sun. Despite the distance, the Sun has a significantly large mass that makes the Sun-EarthMoon network a system of large influence between the parts. The 


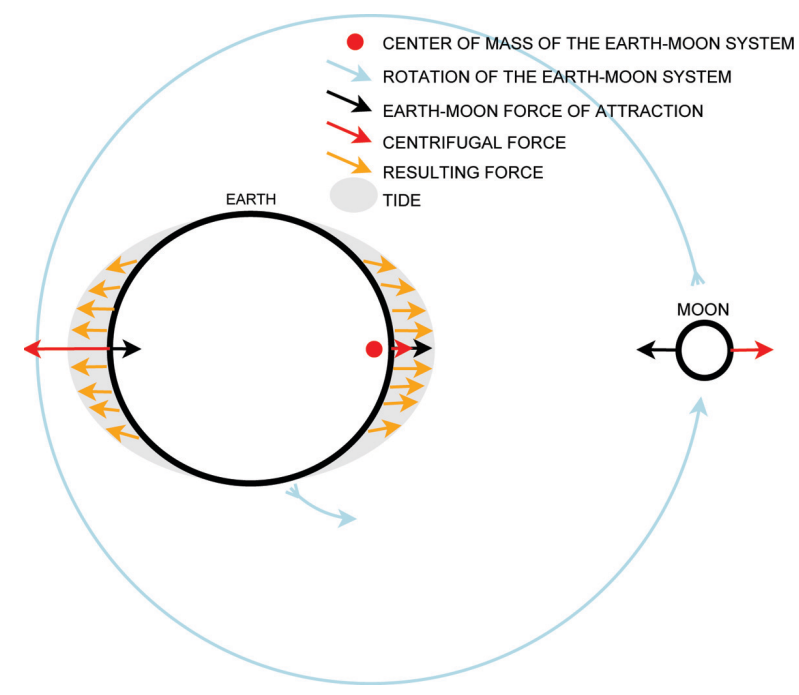

Figure 8 - Tide. Figure adapted from MacMillan (1966).

Earth-Moon system spins around a common center of mass near the Earth because the mass of the Moon is smaller (approximately 1/81 times the Earth's mass). Because the Earth-Moon system spins around a common center of mass between the heavenly bodies, the centrifugal force is small on the terrestrial face closest to the Moon, and it is added to the attractive force; on the opposite side of the Earth, the centrifugal force opposes the force of attraction and is equilibrated, also creating a high tide on the opposite side of the planet, as observed in Figure 8 (Pugh, 1987).

The center of mass, also known as the centroid, is between the heavenly bodies at approximately $1,710 \mathrm{~km}$ beneath the terrestrial surface (Xie \& Kopeikin, 2010).

Tides oscillate on a period of approximately 12 hours and 24 minutes. The "12 hours" are due to the rotation of the Earth, and the "24 minutes" are due to the daily delay caused by the lunar orbit, which has a cycle of 29.5 days. The phenomenon known as the syzygy tide occurs when the Earth, Sun, and Moon are in the same alignment (Full Moon and New Moon). During this period, the variation between the high tide and the low tide is the largest. The quadrature tide occurs when the Moon is waxing or waning, phases in which the variations are smoother. The differences in the tide that occur at the syzygy tide and the quadrature tide are presented in Figure 9.

\section{CALCULATION OF THE TIDE CORRECTION}

In this section, the schematization of the operations necessary for calculating the tide correction, also known as the gravimetric cor- rection (Gemael, 1985), will be presented to permit the codification of a program to calculate the lunar-solar attraction at a specific point $P$ on the surface of the Earth at time $t$ with the use of the formulas presented by Longman (1959) and adjusted using the gravimetric factor. According to Dehlinger (1978), the formulas presented by Longman (1959) are essentially identical to those proposed by Bartels (1957), Schureman (1940), and Pettit (1954).

The calculation of the tide correction $C_{g}$ of a point $P$ on the surface of the Earth is given by (Gemael, 2002):

$$
C_{g}=\left(g_{\odot}+g_{L}\right) \delta
$$

where:

$g_{L}$ is the lunar component of the tide defined by Eq. (14);

$g_{\odot} \quad$ is the solar component of the tide defined by Eq. (37);

$\delta \quad$ is the gravimetric factor.

The gravimetric factor is used to adjust the gravimetric correction value as a function of the displacement of the point $P$ that occurs due to deformation of the crust, which is not solid and has a certain deformation as a function of the lunar-solar attraction. The commonly used programs in Brazil do not allow a user to insert the value of the gravimetric factor and adopt fixed factors, as is the case of REDGRAV ${ }^{5}$, which always uses $\delta=1.20$, and GRAVSYS $^{6}$, which uses $\delta=1.16$.

Technological advancements, the creation of more precise instruments, automated data storage, and the regionalization of the gravimetric factor necessitates the construction of a new software technology to meet the current reality of gravimetric surveys. It is

\footnotetext{
${ }^{5}$ REDGRAV: Software for processing raw gravity data developed by the Universidade de São Paulo, Brazil.

${ }^{6}$ GRAVSYS: Computer program for processing and adjustment of gravimetric data developed at the GSC (Geological Survey of Canada) in Ottawa.
} 


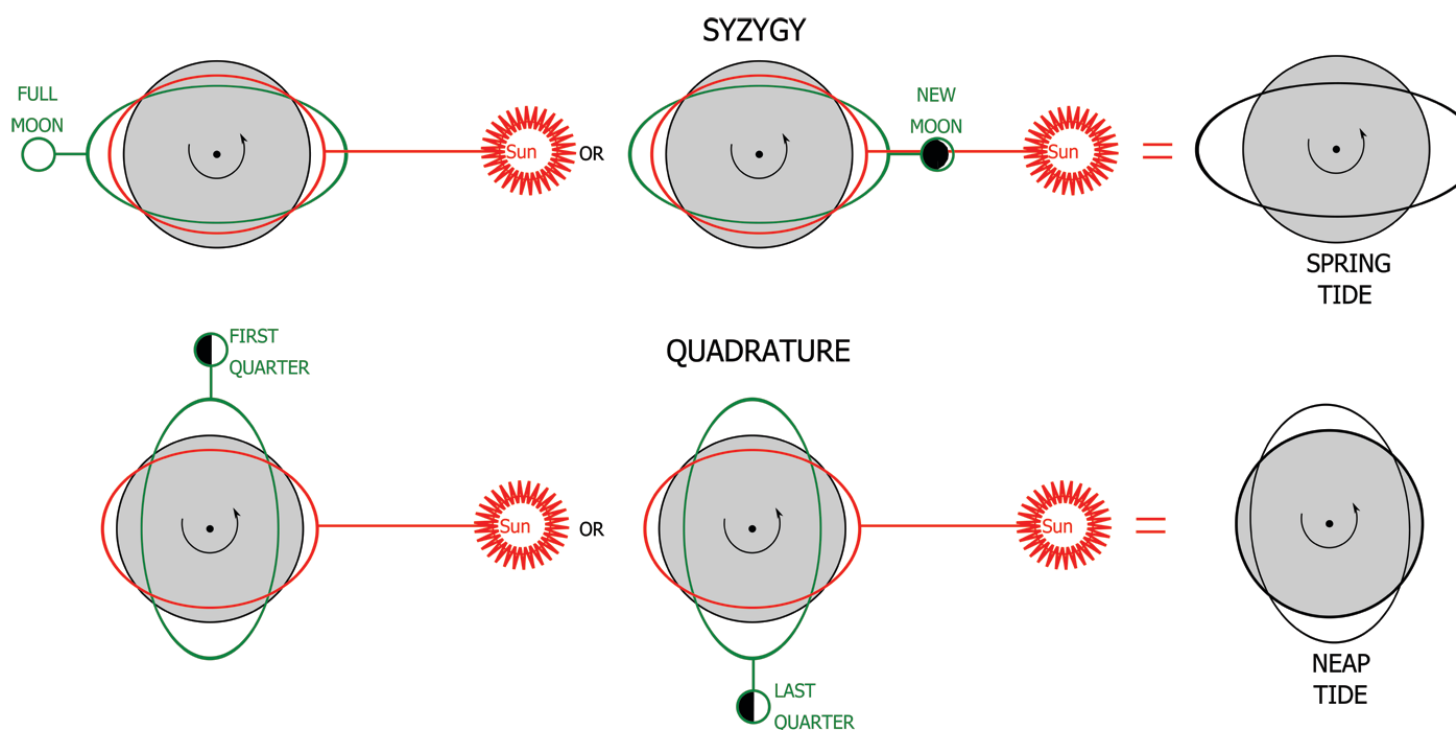

Figure 9 - Variation of the tide amplitude. Figure adapted from Pugh (1987).

easy to find studies that seek to better determine the gravimetric factor in various regions of the world, such as the study by Hassan et al. (2010). For a long time, gravimetric campaigns used the value of $\delta=1.2$ for the gravimetric factor throughout Brazil. However, in a study presented in 1991, regionalization of the gravimetric factor was performed as presented in Table 1. In the present study, tests were conducted with the tide, which are available in section "EVALUATION OF RELEVANCE".

Table 1 - Regionalization of the gravimetric factor (Gemael, 2002).

\begin{tabular}{|l|c|}
\hline Station & $\delta$ \\
\hline Belém & 1.19 \\
Manaus & 1.19 \\
Teresina & 1.22 \\
Caicó & 1.23 \\
Salvador & 1.19 \\
Goiânia & 1.16 \\
Cuiabá & 1.16 \\
Campo Grande & 1.17 \\
Viçosa & 1.20 \\
Vassouras & 1.19 \\
Presidente Prudente & 1.17 \\
Curitiba & 1.17 \\
Santa Maria & 1.20 \\
\hline
\end{tabular}

For codification in the computer, the sequence shown in the mathematical form was altered so that it was possible to implement it in a programming language because the execution sequence of the commands is essential for code functioning. For the trigonometric calculations, angular values were transformed into radians to make them compatible with the most commonly used programming languages. The final version tested is used to develop the software GravSur ${ }^{7}$ (Amarante, 2012).

During the development of this study, some values suggested by Longman (1959) were exchanged for constants defined in more recent investigations, such as the mass of the Sun reported by the documentation of IERS Conventions (International Earth Rotation and Reference Systems Service Central Bureau) (Petit \& Luzum, 2010). Some of these constants, despite having their values reconfigured, do not significantly alter the result; however, with the advance of equipment that can determine differences in the measurements with more precision, it is also necessary to review values that may have been calculated with a slide rule.

The time zone is critical for calculating the tide, and much confusion can occur when determining the time, which can significantly alter the result for the tide correction. This confusion often occurs when the difference that is used for UTC (Coordinated Universal Time) is not recorded or when the team that makes the field measurements is not the same as the one that performs the calculations.

Another important problem is that the official time, which changes as a function of legislation, as is the case of Law No. 11,662 (Brasil, 2008) presented in Figure 10, which modified the boundaries of the regions and the number of time zones in Brazil, as well as the data from the beginning and end of daylight savings time. These changes in the rules for calculating the

\footnotetext{
${ }^{7}$ GravSur: Computer program for processing and adjustment of gravimetric data developed at the Universidade Estadual de Campinas and Universidade de São Paulo.
} 


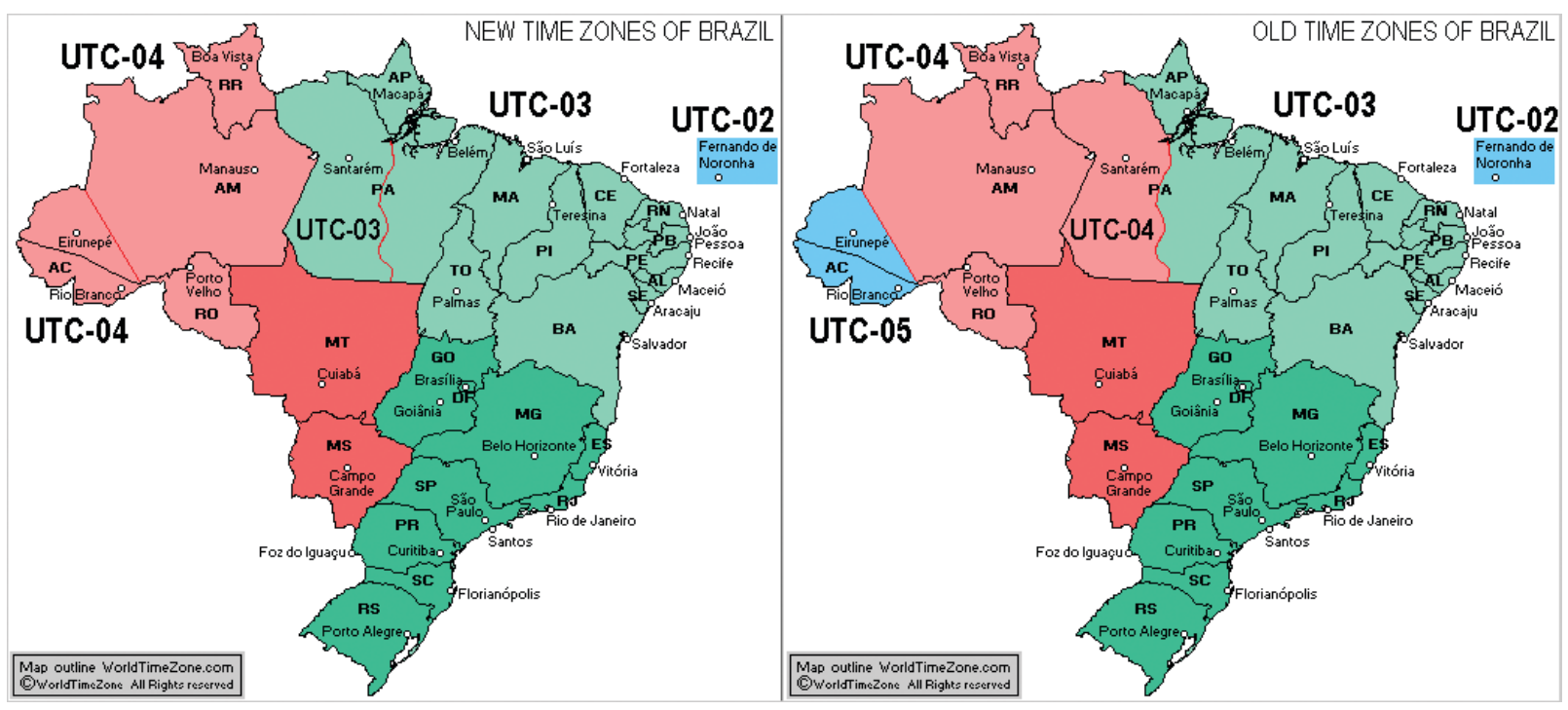

Figure 10 - Change in the time zones in Brazil in 2008 (Worldtimezone, 2011).

official time can cause an incorrect choice when the calculation is performed at some time after the fieldwork. To avoid problems of this type, a protocol can be adopted, as was adopted in civil aviation, which determines the use of the UTC times.

The influence of the Sun can reach approximately $0.063 \mathrm{mGal}$, while that of the Moon can reach up to $0.168 \mathrm{mGal}$ (Gemael, 2002); these values must be considered because they are far above the $0.01 \mathrm{mGal}$ resolution of gravimeters such as the L\&R model $\mathrm{G}$, which are frequently used in campaigns in Brazil and Latin America. Currently, these machines are being replaced by more advanced models, such as the CG-5 Autograv gravimeter from the Scintrex brand with a resolution of $0.001 \mathrm{mGal}$ or $1 \mu \mathrm{Gal}$, which is even more precise. Venus, with a mass of approximately $4.8685 \times 10^{24} \mathrm{~kg}$, can reach a minimum distance of $38,200,000 \mathrm{~km}$ (Williams, 2010) and thus affects the tide by up to $0.00862 \mu$ Gal. Jupiter, with its large mass of $1.8987 \times 10^{30} \mathrm{~kg}$, reaches a nearest distance of 588,500,000 km (Williams, 2012), which has an influence of up to $0.00092 \mu \mathrm{Gal}$. Mars, with its approximate mass of $6.419 \times 10^{23} \mathrm{~kg}$ (Beatty et al., 1999), can reach a minimum distance of 55,760,000 km (McKim, 2003) and can thus cause an influence of up to $0.00037 \mu \mathrm{Gal}$, as demonstrated in Eqs. (12) and (13). Therefore, it is still not necessary to consider the most significant close heavenly bodies such as the planets Venus, Jupiter, and Mars when calculating the tide because these planets' influences can achieve values well below the resolutions of the (relative) pieces of equipment, as CG-5, currently in use.

The influence on the tidal correction due to a heavenly body is given by (Gemael, 2002):

$$
g_{i}=\frac{G M_{i} r_{i}}{\varsigma^{3}}\left(3 \cos ^{2} \theta_{i}-1\right) \delta
$$

adopting the values:

$$
\begin{aligned}
G= & 6.67428 \times 10^{-8} \mathrm{~m}^{3} \mathrm{~g}^{-1} \mathrm{~S}^{-2} \quad \text { (Petit \& } \\
& \text { Luzum, 2010); } \\
g_{i}= & \text { influence of the heavenly body on the tide de- } \\
& \text { fined by Eq. (12); } \\
M_{\odot}= & 4.8685 \times 10^{27} \mathrm{~g} \text { (Venus mass); } \\
M_{4}= & 1.8987 \times 10^{30} \mathrm{~g} \text { (Jupiter mass); } \\
M_{\sigma^{\top}}= & 6.4190 \times 10^{26} \mathrm{~g} \text { (Mars mass); } \\
r_{i}= & 6.378137 \times 10^{8} \mathrm{~cm} ; \\
\varsigma_{\odot}= & 3.82 \times 10^{12} \mathrm{~m} \text { (Venus); } \\
\varsigma_{4}= & 5.8850 \times 10^{13} \mathrm{~m} \text { (Jupiter); } \\
\varsigma_{\sigma^{\top}}= & 5.5760 \times 10^{12} \mathrm{~m} \text { (Mars); } \\
\theta_{i}= & 0^{\circ} \text { to simulate the largest influence; } \\
\delta= & 1.17 \text { for the region of Presidente Prudente } \\
& \text { (Gemael, 2002); }
\end{aligned}
$$

with:

$G$ is Newton's gravitational constant;

$M_{i} \quad$ is the mass of the perturbing heavenly body in grams

$$
\left(M_{\varphi} \text { or } M_{4} \text { or } M_{\sigma^{x}}\right) \text {; }
$$

$r_{i} \quad$ is the distance $P$ in $\mathrm{cm}$ to the center of the Earth;

$\varsigma \quad$ is the maximum approximation of the perturbing heavenly body ( $\varsigma_{0}$ or $\varsigma_{4}$ or $\left.\varsigma_{\mathrm{O}^{\prime}}\right)$;

$\theta_{i} \quad$ is the zenith angle of the perturbing heavenly body;

$\delta \quad$ is the gravimetric factor.

Considering the example of the perihelion opposition of Mars 
in August 2003, when it is very close to the Earth, the influence of the planet in the tide can reach $0.37 \mathrm{nGal}$. Applying similar conditions to Jupiter and Venus has respectively values of $0.92 \mathrm{nGal}$ and $8.62 \mathrm{nGal}$.

The calculation of the tide correction $C_{g}$ of a point $P$ on the surface of the Earth, considering next most significant planets is given by:

$$
C_{g}=\left(g_{\odot}+g_{L}+g_{\odot}+g_{4}+g_{\bigcirc^{\pi}}+\cdots\right) \delta
$$

with:

$g_{\text {}}$ is the Venus component of the tide Eq. (12);

$g_{4}$ is the Jupiter component of the tide Eq. (12);

$g_{\bigcirc^{\top}} \quad$ is the Mars component of the tide Eq. (12).

\section{Lunar Component}

The lunar component $g_{L}$ is giving by:

$$
\begin{aligned}
g_{L}= & \frac{G M_{L} r}{d_{L}^{3}}\left(3 \cos ^{2} \theta_{L}-1\right) \\
& +\frac{3}{2} \frac{G M_{L} r^{2}}{d_{L}^{4}}\left(5 \cos ^{3} \theta_{L}-3 \cos \theta_{L}\right)
\end{aligned}
$$

adopting the values:

$$
\begin{array}{ll}
M_{L}= & 7.34581119761 \times 10^{25} \mathrm{~g} \text {, source: IERS } \\
& \text { data (Petit \& Luzum, 2010). } \\
\text { where: } & \text { is the Newton's gravitional constant; } \\
G & \text { is the Mass of the Moon in grams; } \\
M_{L} & \text { is the distance } P \text { to the center of the Earth defined } \\
& \text { in the Eq. (15); } \\
d_{L} & \text { is the distance between the centers of the Earth and } \\
& \text { the Moon, as defined by the Eq. (19); } \\
\theta_{L} & \text { is the zenith angle of the Moon, shown in Eq. (24). }
\end{array}
$$

The distance $P$ to the center of the Earth is given by:

$$
r=C a+100 H
$$

adopting the value:

$a=6.378137 \times 10^{8} \mathrm{~cm}$ SIRGAS 2000 reference system GRS80 ellipsoid (IBGE, 2005);

where:

$C$ is the value defined in Eq. (16);

$a$ is the equatorial radius of the Earth;

$H$ is the orthometric height (multiplication by 100 is for the transformation into $\mathrm{cm}$ ).

The Earth's equatorial radius of $a=6,378,270$, shown in Longman (1959) and used by REDGRAV, refers to the 1906
Hough ellipsoid, while GRAVSYS uses $a=6,378,388$ from Hayforf (1924). Only updating this value for the GRS80 ellipsoid in a future program is not interesting because the same application can be used in other seasons and regions of the world, where these values can be different. Equation 16 for the calculation of $C$, Longman (1959), uses the constant 0.006738 default in place of $e_{e}^{\prime 2}$, which is only appropriate for the Hough Datum (1906).

$$
C=\sqrt{1 /\left(1+e_{e}^{\prime 2} \sin ^{2} \varphi\right)}
$$

where:

$$
\begin{gathered}
e_{e}^{\prime 2}=\frac{a^{2}-b^{2}}{b^{2}} \\
b=a-(a f)
\end{gathered}
$$

adopting the value:

$$
\begin{aligned}
f= & 1 / 298.257222101 \text { SIRGAS 2000 reference system } \\
& \text { GRS80 ellipsoid (IBGE, 2005); }
\end{aligned}
$$

with:

$\varphi \quad$ is the latitude from point $P$ in decimal degrees;

$e_{e}^{\prime 2}$ is the square of the second eccentricity of the ellipsoid;

$b$ is the minor semiaxis;

$f \quad$ is the flattening of the ellipsoid.

Calculation of the distance $d_{L}$ between the centers of the Earth and the Moon is obtained with:

$$
\begin{aligned}
d_{L}^{-1}= & 1 / c+a^{\prime} e \cos (s-p)+a^{\prime} e^{2} \cos 2(s-p) \\
& +(15 / 8) a^{\prime} m e \cos \left(s-2 h_{1}+p\right) \\
& +a^{\prime} m^{2} \cos 2\left(s-h_{1}\right)
\end{aligned}
$$

adopting the values:

$$
\begin{array}{ll}
c & =3.844031 \times 10^{10} \mathrm{~cm} ; \\
e & =0.05490(\text { Schureman, 1940); } \\
m & =0.074804 \text { (Schureman, 1940); }
\end{array}
$$

with:

$c$ is the average distance between the centers of the Earth and the Moon;

$e \quad$ the eccentricity of the orbit of the Moon;

$a^{\prime}$ is defined by Eq. (20);

$s$ is the average longitude of the Moon referring to the equinox (Bartels, 1957);

$p \quad$ is the average longitude of the perigee of the Moon defined by Eq. (23);

$m$ is the average rate of movement of the Sun to the Moon

where:

$$
a^{\prime}=1 /\left[c\left(1-e^{2}\right)\right]
$$


To determine $s$ and $p$, it is first necessary to know the number of Julian "centuries" $T_{j}$ since December 31,1899 , which is calculated with:

$$
T_{j}=t / 52,596,000
$$

with:

$t \quad$ is the instant of determination of the tide in the Julian calendar;

$T_{j} \quad$ adopting a Julian century as (100 years $\cdot 365.25$ days . $24 \mathrm{hr} \cdot 60 \mathrm{~min})=52,596,000$ minutes.

The average longitude of the Moon referring to the equinox is given by:

$$
\begin{aligned}
s= & 270^{\circ} 26^{\prime} 11.72^{\prime \prime} \\
& +\left(1336 r e v .+1,108,406.05^{\prime \prime}\right) T_{j} \\
& +7.128^{\prime \prime} T_{j}^{2}+0.0072^{\prime \prime} T_{j}^{3}
\end{aligned}
$$

For the calculation of the average longitude of lunar perigee, the following equation is used:

$$
\begin{aligned}
p= & 334^{\circ} 19^{\prime} 46.42 \\
& +\left(11 \text { rev. }+392,522.51^{\prime \prime}\right) T_{j} \\
& -37.15^{\prime \prime} T_{j}^{2}-0.036^{\prime \prime} T_{j}^{3}
\end{aligned}
$$

The REDGRAV program uses the eccentricity of the lunar orbit suggested by Shureman (1924); however, this study uses a new value given by the 1940 revision of the book (Schureman, 1940). Despite this difference, the calculation does not yield significant results. One purpose of this paper is to help in the implementation of algorithms in future gravimetric data processing programs. Therefore, the values of $s, p, h_{\odot}$ and all other variables that represent angles from equations suggested in Longman (1959) must be transformed into radians to permit the use of the computational functions. The abbreviation rev., shown in Eqs. (22) and (23), refers to revolutions; that is, each unit must be multiplied by $360^{\circ}$.

The zenith angle of the Moon $\theta_{L}$ is determined by:

$$
\begin{gathered}
\cos \theta_{L}=\sin \varphi \sin I \sin l \\
+\cos \varphi\left[\cos ^{2} \frac{I}{2} \cos (l-\chi)+\sin ^{2} \frac{I}{2} \cos (l+\chi)\right]
\end{gathered}
$$

with:

$I$ is the angle between the orbit of the Moon and the celestial equator according to (Fig. 11) defined by Eq. (25); $l \quad$ is the longitude of the Moon in its orbit counted from the crossing of the ascension with the equator line defined by Eq. (31);

$\varphi \quad$ is the latitude of point $P$;

$A$ is the ascending intersection of the lunar orbit with the equator;

$\chi \quad$ is the ascension to the right of the meridian of observation counted from $A$ (Fig. 11), obtained using Eq. (27);

$i \quad$ is the constant given by Schureman (1940) used in Eq. (28) referring to the angle between the lunar orbit and the plane of the ecliptic (Fig. 11).

Determination of the angle $I$ between the lunar orbit and the celestial equator is given by:

$$
\cos I=\cos \omega_{t} \cos i-\sin \omega_{t} \sin i \cos N_{\lambda}
$$

adopting the value:

$$
i=5^{\circ} 08^{\prime} 43.3546^{\prime \prime}
$$

The longitude $N_{\lambda}$ from the ascending node to the orbit of the Moon is defined by:

$$
\begin{aligned}
N_{\lambda}= & 259^{\circ} 10^{\prime} 57.12^{\prime \prime} \\
& -\left(5 \mathrm{rev} .+482,912.63^{\prime \prime}\right) T_{j} \\
& +7.58^{\prime \prime} T_{j}^{2}+0.008^{\prime \prime} T_{j}^{3}
\end{aligned}
$$

and $\chi$ is given by:

$$
\chi=t_{a}+h_{\odot}-v
$$

where:

$$
v=\arcsin (\sin i \sin N / \sin I)
$$

The angular time $t_{a}$ of the average measurement of the Sun to the west of the location of observation is defined by:

$$
t_{a}=15\left(t_{0}-12\right)-\lambda
$$

Average longitude $h_{\odot}$ from the Sun is given by:

$$
\begin{aligned}
h_{\odot}= & 279^{\circ} 41^{\prime} 48.05^{\prime \prime} \\
& +129,602,768.11^{\prime \prime} T_{j}+1.080^{\prime \prime} T_{j}^{2}
\end{aligned}
$$

where:

$t_{0}$ is UTC time in whole Julian hours;

$\lambda \quad$ is longitude from the point $P$;

$t_{a}$ is the angular time of the average measurement of the Sun to the west of the location of observation. 


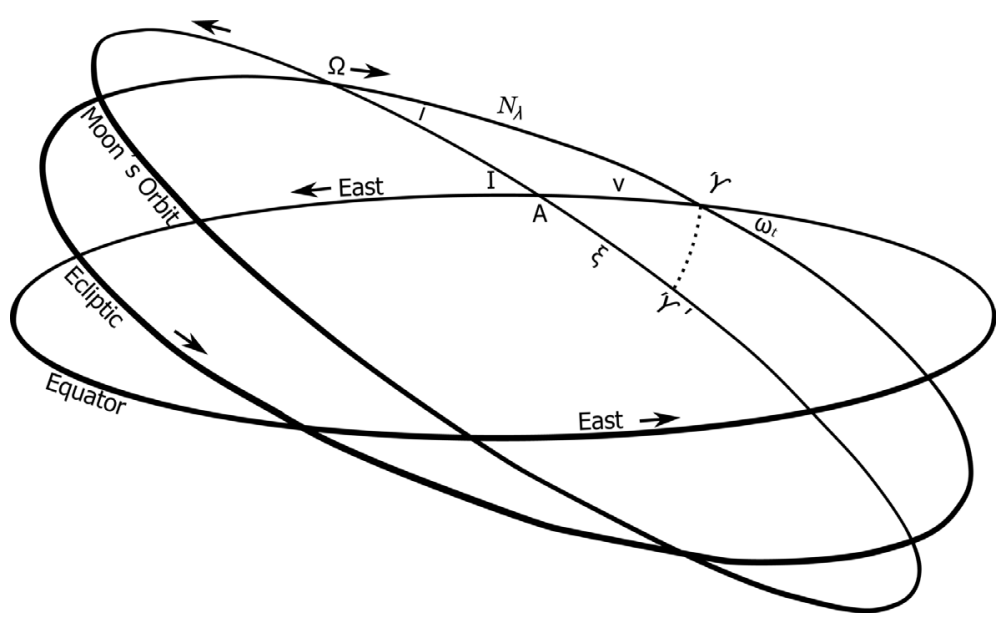

Figure 11 - Orbital parameters (Schureman, 1940).

The longitude of the Moon $l$ is given by:

$$
\begin{aligned}
l= & \sigma+2 e \sin (s-p)+\frac{5}{4} e^{2} \sin 2(s-p) \\
& +\frac{15}{4} m e \sin \left(s-2 h_{1}+p\right) \\
& +1.375 m^{2} \sin 2\left(s-h_{1}\right)
\end{aligned}
$$

with:

$\Upsilon$ is the vernal equinox (Fig. 11);

$\Upsilon^{\prime}$ is the related equinox (Fig. 11);

$\Omega \quad$ is the node from the beginning of the rising of the Moon (Fig. 11);

The average longitude of the Moon $\sigma$ given by the relationship:

$$
\sigma=s-\xi
$$

and the longitude $\xi$ in the intersection of the orbit with the celestial equator is defined by:

$$
\xi=N_{\lambda}-\alpha
$$

where:

$$
\begin{gathered}
\alpha=2 \arctan [\sin \alpha /(1+\cos \alpha)] \\
\sin \alpha=\sin \omega_{t} \sin N_{\lambda} \sin I \\
\cos \alpha=\cos N_{\lambda} \cos v+\sin N_{\lambda} \sin v \cos \omega_{t}
\end{gathered}
$$

\section{Solar component}

The influence of the solar component $g_{\odot}$ on the measurement of the acceleration of a point is determined by Longman (1959):

$$
g_{\odot}=\frac{G M_{\odot} r}{d_{\odot}^{3}}\left(3 \cos ^{2} \theta_{\odot}-1\right)
$$

adopting the values:

$M_{\odot}=1.9884158 \times 10^{33} \mathrm{~g}$, source: IERS data (Petit \& Luzum, 2010);

with:

$M_{\odot}$ is the mass of the Sun in grams;

$d_{\odot} \quad$ is the distance between the centers of the Earth and the Sun, as defined by Eq. (38);

$\theta_{\odot} \quad$ is the zenith angle of the Sun, as defined by Eq. (40).

Calculation of the distance $d_{\odot}$ between the centers of the Earth and the Sun is given by:

$$
\begin{gathered}
d_{\odot}=\frac{1}{c_{\odot}+a_{1}^{\prime} e_{1} \cos \left(h_{\odot}-p_{\odot}\right)} \\
a_{1}^{\prime}=1 /\left[c_{\odot}\left(1-e_{1}^{2}\right)\right]
\end{gathered}
$$

adopting the value:

$c_{\odot}=1.49597870691 \times 10^{13} \mathrm{~cm}$ ) (Seidelmann, 1992);

with:

$c_{\odot} \quad$ is the average distance between the centers of the Earth and the Sun;

$a_{1}^{\prime} \quad$ is defined by Eq. (39);

$e_{1}$ is the eccentricity of the orbit of the Earth;

$h_{\odot} \quad$ is the average longitude of the Sun;

$p_{\odot} \quad$ is the average longitude of solar perigee ${ }^{8}$.

${ }^{8}$ perigee: point in the orbit of an object in which it is closest to the Earth. 
The zenith angle of the $\operatorname{Sun} \theta_{\odot}$ is defined by Equation:

$$
\begin{aligned}
\cos \theta_{\odot}= & \sin \varphi \sin \omega_{t} \sin l_{\odot} \\
& +\cos \lambda\left[\cos ^{2} \frac{\omega_{t}}{2} \cos \left(l_{\odot}-\chi_{\odot}\right)\right. \\
& \left.+\sin ^{2} \frac{\omega_{t}}{2} \cos \left(l_{\odot}-\chi_{\odot}\right)\right]
\end{aligned}
$$

adopting the value:

$$
\omega_{t}=23^{\circ} 27^{\prime} 08.26^{\prime \prime} \text { (Schureman, 1940); }
$$

where:

$\omega_{t}$ is the obliqueness of the ecliptic on January 1, 1900;

$l_{\odot} \quad$ is the longitude of the Sun in the elliptic counted from the Vernal Equinox;

$\chi_{\odot} \quad$ is the ascension to the right of the meridian of observation counted from the Vernal Equinox;

$t_{a}$ is the angular time of the average measurement of the Sun to the west of the location of observation.

The longitude $l_{\odot}$ of the Sun in the elliptic counted from the Vernal Equinox is given by:

$$
l_{\odot}=h_{\odot}+2 e_{1} \sin \left(h_{\odot}-p_{\odot}\right)
$$

The ascension $\chi_{\odot}$ to the right of the meridian of observation counted from the Vernal Equinox is obtained by:

$$
\chi_{\odot}=t_{a}+h_{\odot}
$$

The eccentricity of the Earth's orbit is calculated by:

$$
\begin{aligned}
e_{1}= & 1.675104 \times 10^{-2} \\
& -4.180 \times 10^{-5} T_{j} \\
& -1.26 \times 10^{-7} T_{j}^{2}
\end{aligned}
$$

The average longitude of solar perigee $p_{\odot}$ is defined by:

$$
\begin{aligned}
p_{\odot}= & 281^{\circ} 13^{\prime} 15 \\
& +6,189.03^{\prime \prime} T_{j} \\
& +1.63^{\prime \prime} T_{j}^{2}+0.012^{\prime \prime} T_{j}^{3}
\end{aligned}
$$

\section{METHOD FOR EVALUATION OF RELEVANCE (GRAVIMETRIC FACTOR)}

This study aimed to evaluate the extent to which the calculation of the tide correction with different gravimetric factors is representative. For the gravimetric factor, from a geographical position, the differences between the corrections values of the tide calculated were determined by various programs/machines using the most recommended value for the region, as indicated in (Table 1).
The differences were compared to the resolution of modern devices such as the Scintrex CG-5 and the OSG superconductor gravimeters to calculate the significance of the above-calculated factors.

By comparing the results from the tide calculations generated by the GRAVSYS and REDGRAV with the approach suggested in this study implemented in the GravSur computer program (Amarante, 2012), it was possible to note differences. The REDGRAV program is important because it is used in Brazil and in certain Latin American countries by the EPUSP (Escola Politécnica, Universidade de São Paulo) and IBGE (Instituto Brasileiro de Geografia e Estatística) to calculate the gravimetric reductions.

Table 2 contains some points distributed in the four quadrants of the globe in UCT time; some observations merit consideration. First, GRAVSYS does not allow the input of the year with four digits, which does not permit its use after 1999. A difference is also noted that apparently is not large but becomes evident when the amplitudes of the tides are larger. Notably, GravSur has a configurable gravimetric factor, and the calculation was performed with $\delta=1.17$; by contrast, REDGRAV and GRAVSYS have fixed values of 1.20 and 1.16, respectively. Another important point is that the CG-5 can perform the tide correction automatically; however, it always uses a gravimetric factor of 1.16 , because this value cannot be altered (Scintrex, 2008).

To best illustrate the difference, the example below was calculated at the amplitude peak in a spring (syzygy) tide day:

Location: Santos SP, Brazil

- Position: $23^{\circ} 57^{\prime} \mathrm{S} 046^{\circ} 18^{\prime} \mathrm{W}$

- Date: FEB/18/2011 - 15:20 UCT

- GravSur: 0.188 mGals

- REDGRAV: 0.193 mGals

- GRAVSYS: 0.186 mGals

A difference of up to $0.007 \mathrm{mGals}$ was calculated, which is a significant value because currently used equipment, such as the CG -5 , has a resolution of $0.001 \mathrm{mGals}$. This difference tends to zero when the same gravimetric factor is used; it is emphasized that the value of $\delta=1.17$, referring to the region of Presidente Prudente, is the most appropriate for the city of Santos according to a publication about regionalization of the gravimetric factor from Gemael (2002).

Another important fact related to REDGRAV and GRAVSYS is that neither system considers seconds when recording the time of the reading. Differences of up to $0.001 \mathrm{mGals}$ are observed in 
Table 2 - Comparison of the tide calculation by different programs and local time (mGals).

\begin{tabular}{|c|c|c|c|c|}
\hline Date/Time & Location & GravSur & REDGRAV & GRAVSYS \\
\hline 2010/10/318:10 & $22^{\circ} 44^{\prime} \mathrm{S} 90^{\circ} 30^{\prime} \mathrm{W}$ & -0.055 & -0.056 & - \\
2010/10/318:10 & $22^{\circ} 44^{\prime} \mathrm{S} 90^{\circ} 30^{\prime} \mathrm{E}$ & -0.003 & -0.003 & - \\
2010/10/318:10 & $22^{\circ} 44^{\prime} \mathrm{N} 90^{\circ} 30^{\prime} \mathrm{W}$ & -0.007 & -0.007 & - \\
2010/10/318:10 & $22^{\circ} 44^{\prime} \mathrm{N} 90^{\circ} 30^{\prime} \mathrm{E}$ & -0.052 & -0.053 & - \\
\hline $1996 / 10 / 318: 10$ & $22^{\circ} 44^{\prime} \mathrm{S} 90^{\circ} 30^{\prime} \mathrm{W}$ & 0.028 & 0.029 & 0.028 \\
$1996 / 10 / 318: 10$ & $22^{\circ} 44^{\prime} \mathrm{S} 90^{\circ} 30^{\prime} \mathrm{E}$ & 0.120 & 0.123 & 0.119 \\
$1996 / 10 / 318: 10$ & $22^{\circ} 44^{\prime} \mathrm{N} 90^{\circ} 30^{\prime} \mathrm{W}$ & 0.123 & 0.126 & 0.122 \\
$1996 / 10 / 318: 10$ & $22^{\circ} 44^{\prime} \mathrm{N} 90^{\circ} 30^{\prime} \mathrm{E}$ & 0.029 & 0.030 & -0.029 \\
\hline
\end{tabular}

20-second cycles, which was not very important when using mechanical equipment. Presently, the new types of equipment permit the storage of the time with the assistance of a GPS receiver, which guarantees good quality. Finally, the CG- 5 gravimeter also has a fixed gravimetric factor and, as long as the firmware is not updated to permit configuration of this parameter, in studies where accurate data are sought, the correct approach would be to import data into programs that permit the treatment of the data, as is the case for GravSur.

\section{CONCLUSIONS}

Gravimetry adopts the formulas suggested in the study by Longman (1959) for the gravimetric correction, which was calculated with convenient and known orbital and ellipsoidal parameters for the time. Despite this fact, some computer programs adopt equivalent values, which do not yield significant results where, for example, the ellipsoidal parameters are slightly different.

Superconducting gravimeters can reach sensitivities of $1 \mathrm{nGal}$ (nanogal). The gravimetric correction applied to the processing of data generated from equipment like this should consider the influence of the bodies with significant attractive force. When Venus is in opposition with the Earth can modify the result of gravimetric correction until $8.62 \mathrm{nGal}$.

The gravimetric factor, which can vary according to the region, can represent differences of approximately $0.01 \mathrm{mGal}$ and even so, computer programs such as REDGRAV, GRAVSYS, and current equipment maintain this value as a constant and does not allow set a different factor.

\section{REFERENCES}

AMARANTE RR. 2012. Systematization of gravimetric data processing applied to geoid model determination. Ph.D. Thesis, Universidade Estadual de Campinas, School of Civil Engineering, Architecture and Urban Design, Campinas, São Paulo, Brazil.
BARTELS J. 1957. Geophysik II/ Geophysics II. Handbuch Der Physik Encyclopedia of Physics/ Geophysik/Ge. Springer-Verlag.

BEATTY JK, PETERSEN CC \& CHAIKIN A. 1999. The New Solar System. 4th ed., Sky Publishing Corporation, Cambridge, Massachusetts.

BRASIL. 2008. Lei no. 11.662, 24 de dez. de 2008, Brasília, DF, Brazil.

DEHLINGER P. 1978. Marine Gravity. Vol. 22 of Elsevier Oceanography Series. Elsevier.

GEMAEL C. 1985. Determinação da gravidade geodésica. Imprensa Universitária - UFPR, Universidade Federal do Paraná, Curitiba, Brazil.

GEMAEL C. 2002. Introdução à Geodésica Física. 2nd ed., Editora da UFPR, Curitiba, Paraná, Brazil.

GRW. 2011. GWR Observatory Superconducting Gravimeter. Available on: <http://www.gwrinstruments.com>.

HASSAN RM, ABDELRAHMANEM, TEALEB A, ZAHRAN K \& JENTZSCH G. 2010. Gravimetric Tide observation at Lake Nasser Region, Aswan, Egypt. Marees Terrestres Bulletin d'informations, 146: 11797-11805.

HEGEWALD A, JENTZSCH G \& JAHR T. 2010. Comparison of the LaCoste \& Romberg gravimeter ET18 with the superconducting gravimeter CD-034 at the Geodynamic Observatory Moxa (Germany). Marees Terrestres Bulletin d'informations, 146: 11781-11788.

IBGE - Instituto Brasileiro de Geografia e Estatística. 2005. Resolução 1/2005. Altera a caracterização do Sistema Geodésico Brasileiro, Brazil.

LACOSTE \& ROMBERG. 2004. Instruction Manual Model G\&D Gravity Meters, Austin, $127 \mathrm{pp}$.

LONGMAN IM. 1959. Formulas for computing the tidal accelerations due to the Moon and the Sun. Journal of Geophysical Research, 64(12): 2351-2355.

MacMILLAN DH. 1966. Tides. C R Books Limited, London, UK.

McKIM R. 2003. The great perihelic opposition of mars, 2003: Part 1. Journal of the British Astronomical Association, 120: 280-295.

MORITZ H. 1980. Geodetic reference system 1980. Bulletin Géodésique, 54: 395-405. 
PETTIT JT. 1954. Tables for the computation of the tidal accelerations of the Sun and Moon. Vol. 35. Transactions American Geophysical Union.

PETIT G \& LUZUM B. 2010. IERS Conventions (2010). Verlag, Germany.

PUGH DT. 1987. Tides, Surges and Mean Sea-Level. John Wiley \& Sons Ltd., Natural Environment Research Council Swindon, UK ISBN $047191505 X$.

SCHUREMAN P. 1940. Manual of harmonic analysis and prediction of tides. No. 98. U.S. Department of Commerce. Special Publication.

SCINTREX. 2008. CG-5 Scintrex Autograv System operation Manual. 4th ed.

SEIDELMANN PK. 1992. Explanatory supplement to the Astronomical Almanac. University Science Books.

SI. 2006. The International System of Units. Bureau International des
Poids et Mesures, 8th ed.

TORGE W. 2001. Geodesy. 3rd ed., Walter de Gruyter.

WILLIAMS DR. 2010. Nasa Venus Fact Sheet. Available on: < http:// nssdc.gsfc.nasa.gov/planetary/factsheet/venusfact.html>.

WILLIAMS DR. 2012. Nasa Jupiter Fact Sheet. Available on: < http:// nssdc.gsfc.nasa.gov/planetary/factsheet/jupiterfact.html>.

WORLDTIMEZONE. 2011. New Brazil time zones map and old Brazil time zones map. Available on: <http://www.worldtimezone.com/braziltime-new-old.php>.

XIE Y \& KOPEIKIN S. 2010. Post-Newtonian Reference Frames for Advanced Theory of the Lunar Motion and for a New Generation of Lunar Laser Ranging. Acta Physica Slovaca. Reviews and Tutorials, 60: 393-495. 